\title{
Analyses of multiplicity distributions and Bose-Einstein correlations at the LHC by means of generalized Glauber-Lachs formula
}

\author{
Takuya Mizoguchi* \\ Toba National College of Maritime Technology, Toba 517-8501, Japan \\ E-mail: mizoguti@toba-cmt.ac.jp \\ Minoru Biyajima \\ Department of Physics, Shinshu University, Matsumoto 390-8621, Japan \\ E-mail: biyajima@azusa.shinshu-u.ac.jp
}

\begin{abstract}
Using the negative binomial distribution (NBD) and the generalized Glauber-Lachs (GGL) formula, we analyze the data on charged multiplicity distributions in the several pseudorapidity intervals $|\eta|<\eta_{c}$ at $0.2-7 \mathrm{TeV}$ by UA5 and ALICE Collaborations. We confirm that the KNO scaling holds among the multiplicity distributions with $\eta_{c}=0.5$ at $\sqrt{s}=0.2-2.36 \mathrm{TeV}$ and estimate the energy dependence of a parameter $1 / k$ in NBD and parameters $1 / k$ and $\gamma$ (the ratio of the average value of the coherent hadrons to that of the chaotic hadrons) in the GGL formula. Using empirical formulae for the parameters $1 / k$ and $\gamma$ in the GGL formula, we predict the multiplicity distributions with $\eta_{c}=0.5$ at 7 and $14 \mathrm{TeV}$. Data on the second order Bose-Einstein correlations (BEC) at 0.9 and $2.36 \mathrm{TeV}$ by ALICE and CMS Collaborations are also analyzed based on the GGL formula. Predictions for the third order BEC at 0.9 and $2.36 \mathrm{TeV}$ are presented.
\end{abstract}

The Seventh Workshop on Particle Correlations and Femtoscopy

September 20 - 242011

University of Tokyo, Japan

\footnotetext{
*Speaker.
} 


\section{Introduction}

Recently ALICE Collaboration [1] has investigated the multiplicity distributions with pseudorapidity cutoffs and compared its data with the data by UA5 Collaboration [2, 3], and concluded that the combined data with $\eta_{c}=0.5$ at $0.2,0.9$, and $2.36 \mathrm{TeV}$ are fairly well described by the single NBD (negative binomial distribution) [4, 5]. Moreover, ALICE Collaboration has reported that the KNO scaling [6] holds among the combined data with $\eta_{c}=0.5$ at $0.2,0.9$, and $2.36 \mathrm{TeV}$. The first aim of this study is to confirm the statement above mentioned in [1] and to analyze the same data by the GGL(generalized Glauber-Lachs) formula [7, 8]. Some predictions at $2.36 \mathrm{TeV}[9]$ are also included in this proceeding.

Moreover, ALICE and CMS Collaborations have reported the data on Bose-Einstein correlations (BEC) [10, 11]. Thus we investigate them based on a conventional formula with the degree of coherence and the GGL formula. According to main results in Ref. [12], our talk is presented.

The NBD is introduced in the following:

$$
P_{k}(n)=\frac{\Gamma(n+k)}{\Gamma(n+1) \Gamma(k)} \frac{(\langle n\rangle / k)^{n}}{(1+\langle n\rangle / k)^{n+k}},
$$

where $\langle n\rangle$ and $k$ are the average multiplicity and the intrinsic parameter, respectively. In the KNO scaling limit ( $n$ and $\langle n\rangle$ are large, but the ratio $z=n /\langle n\rangle$ is finite), for the quantity $\langle n\rangle P(n,\langle n\rangle)$ the following gamma distribution is derived from Eq. (1.1) as

$$
\psi_{k}(z)=\frac{k^{k}}{\Gamma(k)} z^{k-1} e^{-k z}
$$

Second we turn to the GGL formula which is expressed as follows:

$$
P_{k}(n)=\frac{(p\langle n\rangle / k)^{n}}{(1+p\langle n\rangle / k)^{n+k}} \exp \left[-\frac{\gamma p\langle n\rangle}{1+p\langle n\rangle / k}\right] L_{n}^{(k-1)}\left(-\frac{\gamma k}{1+p\langle n\rangle / k}\right),
$$

where $\gamma=|\zeta|^{2} / A$ (the ratio of the average value of the coherent hadrons to that of the chaotic hadrons), $p=1 /(1+\gamma)$, and $L_{n}^{(k-1)}$ stands for the Laguerre polynomials, respectively.

The KNO scaling function of Eq. (1.3) is given in the following

$$
\psi_{k}(z, p)=\left(\frac{k}{p}\right)^{k}\left[\frac{z}{\sqrt{z(k / p)^{2}(1-p)}}\right]^{k-1} \exp \left[-\frac{k}{p}(1-p+z)\right] I_{k-1}\left(2 \sqrt{z(k / p)^{2}(1-p)}\right)
$$

where $I_{k-1}$ is the modified Bessel function. Eq. (1.4) becomes the gamma distribution, as $\gamma=0$.

In order to analyze of Bose-Einstein correlations (GGLP effect [13], or hadronic HBT effect [14, 15]) at LHC, we are going to use the following formulae: The first one is well known as the conventional formula,

$$
\begin{aligned}
& N^{(--)} / N^{B G}(\text { conventional formula })=c\left[1+\lambda E_{2 B}^{2}\right], \\
& N^{(--)} / N^{B G}(\mathrm{GGL})=c\left[1+2 p(1-p) E_{2 B}+p^{2} E_{2 B}^{2}\right]
\end{aligned}
$$

where $c$ is normalization factor, $\lambda$ is the degree of coherence, $p=1 /(1+\gamma)$ and $E_{2 B}$ is function of momentum transfer $\left(Q^{2}=-\left(p_{1}-p_{2}\right)^{2}\right)$ and the range of interaction $R . E_{2 B}=\exp \left(-R^{2} Q^{2}\right)$ (Gaussian formula) and/or $E_{2 B}=\exp \left(-R \sqrt{Q^{2}}\right)$ (exponential formula) are used. 


\section{Analyses of data on multiplicity distributions by the NBD and the GGL formula}

Utilizing Eqs. (1.1) and (1.3), we analyze the data with pseudo-rapidity cutoffs $\left(\eta_{c}=0.5,1.0\right.$, and 1.3) at $0.2,0.54,0.9$ and $2.36 \mathrm{TeV}$. Results at 0.9 and $2.36 \mathrm{TeV}$ are shown in Fig. 1, Energy
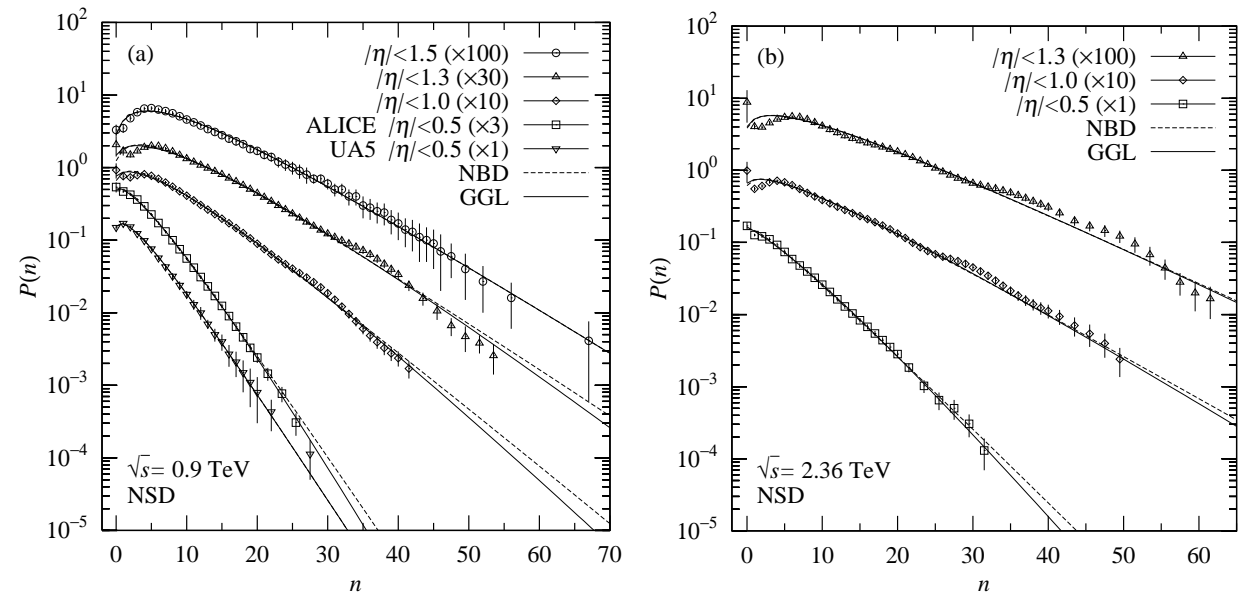

Figure 1: Analyses of data with $|\eta|<\eta_{c}$ by means of Eqs. (1.1) and (1.3). (See estimated values of parameters contained in Eqs. (1.1) and (1.3) shown in Fig. 2 and [12]).

dependences of parameters $1 / k^{(\mathrm{NBD})}, 1 / k^{(\mathrm{GGL})}$ and $\gamma^{(\mathrm{MD})}$ (MD: multiplicity distribution) with $\eta_{c}=$ 0.5 are shown in Fig. 2. We observe that $1 / k^{(\mathrm{NBD})}$ increases gradually as $\sqrt{s}$ increases. On the other hand, the estimated sets of $\left(1 / k^{(\mathrm{GGL})}\right.$ and $\left.\gamma^{(\mathrm{MD})}\right)$ in the GGL formula show different behavior.

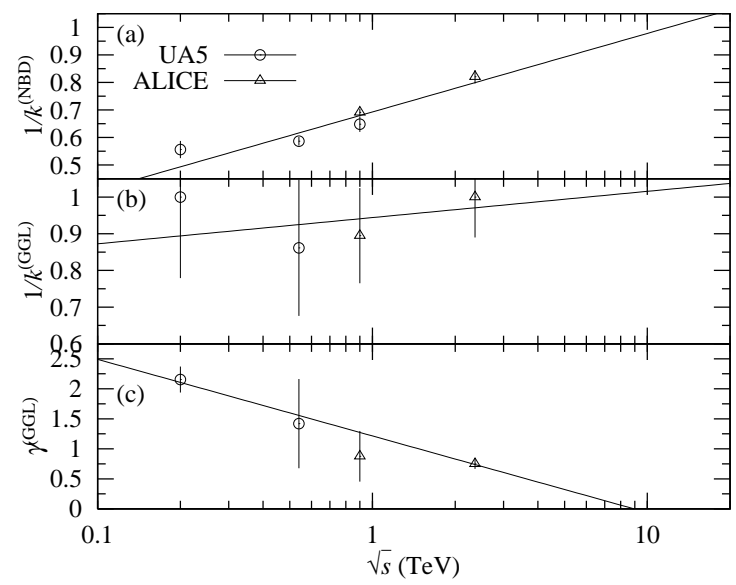

Figure 2: Energy dependences of parameters $1 / k^{(\mathrm{NBD})}, 1 / k^{(\mathrm{GGL})}$, and $\gamma^{(M D)}$ for data with $\eta_{c}=0.5$. Values at $0.9 \mathrm{TeV}$ by UA5 Collaboration are omitted in GGL formula, because of extreme error bars.

\section{Analyses of data on KNO scaling distributions by Eqs. (1.2) and (1.4)}

Utilizing the KNO scaling variable $z(=n /\langle n\rangle)$, data on the KNO scaling distributions $\langle n\rangle P(n,\langle n\rangle)$ are shown in Fig. 3 . We combine the data with $\eta_{c}=0.5$ at $0.2,0.9$ and $2.36 \mathrm{TeV}$ and analyze them by Eq. (1.2) (the gamma distribution) and Eq. (1.4) (the modified Bessel function).

\section{Analyses of data on the 2nd order BEC by means of Eqs. (1.5) and (1.0)}

We analyze the data on BEC at LHC by the use of Eqs. (1.5) and (1.6) with $E_{2 B}=\exp \left(-R^{2} Q^{2}\right)$ and/or $E_{2 B}=\exp \left(-R \sqrt{Q^{2}}\right)$. Results are depicted in Table ${ }_{1}$ and Fig. ${ }_{\text {T. }}$ In Eq. (1.6) the effec- 

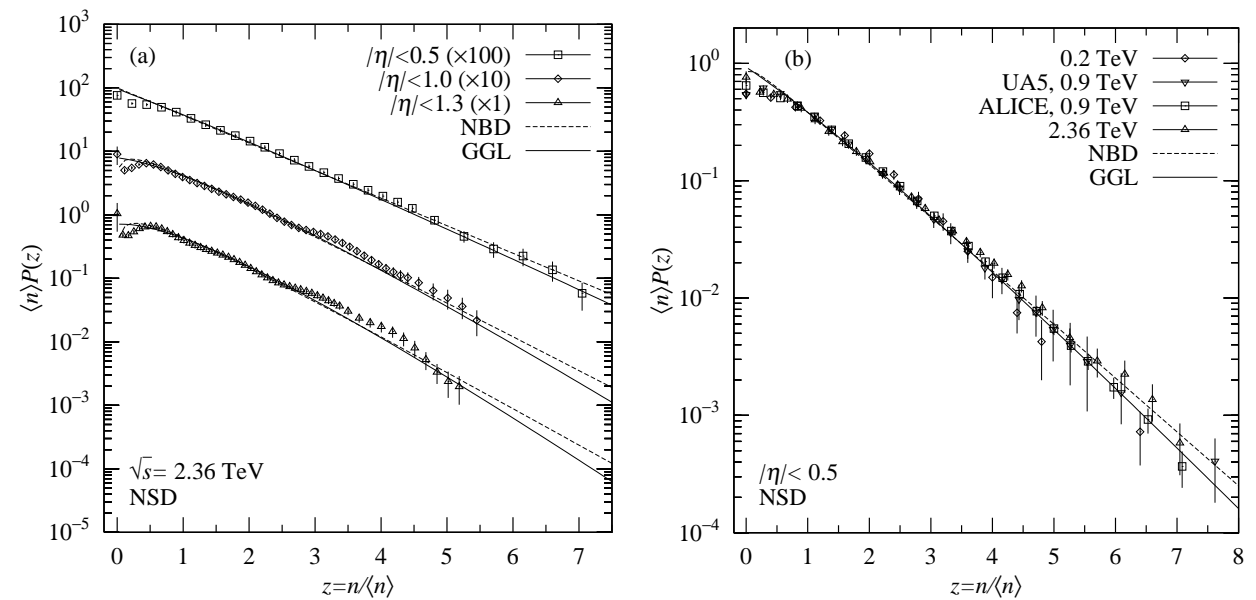

Figure 3: Analyses of KNO scaling distributions $\langle n\rangle P(n)$ 's. The same data of Fig. Lare described by KNO scaling variable $z=n /\langle n\rangle$. Eqs. (1.2) and (1.4) are used.

tive degree of coherence " $\lambda$ " is " $(1+2 \gamma) /(1+\gamma)^{2}$ ". In our concrete analyses, we obtained that $1 / k^{(\mathrm{BEC})}=1 . \gamma^{(\mathrm{BEC})}$ is similar to the value at $0.9 \mathrm{TeV}$ by ALICE Collaboration in Fig. 2.

Furthermore, by the use of Eqs. (1.5) and (1.6) with $c$, we have analyzed the data on BEC at 0.9 and $2.36 \mathrm{TeV}$ by CMS Collaboration [11]. Results are shown in Fig. 4 and Table $\square$ Notice that estimated values of $\lambda, \gamma$ and $R$ do not depend on the range of exclusive region $(0.4<Q<1.4$ $\mathrm{GeV} / c$ ). It is emphasized that the ratio $\gamma^{(\mathrm{BEC})}$ decreases, as the colliding energy increases. In other words, the effective degree of coherence " $\lambda$ " and the range of interaction $R$ increases from 0.9 to $2.36 \mathrm{TeV}$. To draw more significant meaning about the parameter $\gamma$, we need BEC measurements with $\eta_{c}=0.5$.
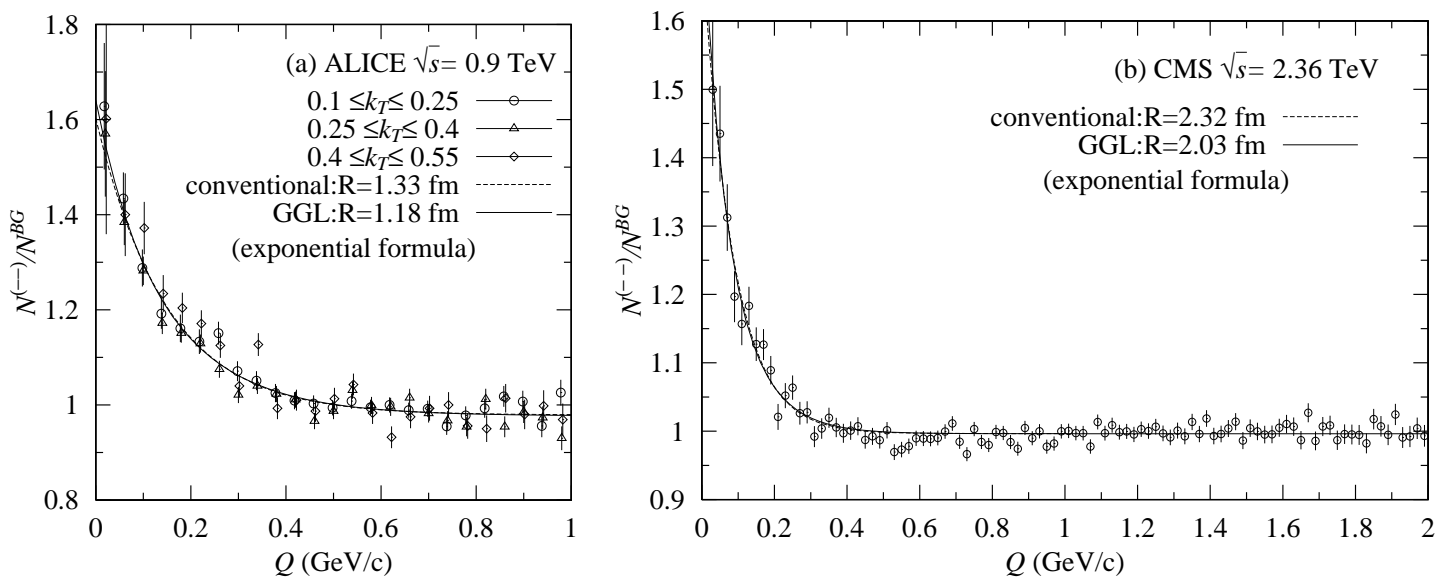

Figure 4: Analyses of data on BEC at $0.9 \mathrm{TeV}$ by ALICE Collaboration with conditions $M \leq 6$, and 0.1 $\leq k_{T} \leq 0.55 \mathrm{GeV}$ and at $2.36 \mathrm{TeV}$ by CMS Collaboration.

\section{Concluding remarks}

We have confirmed that the multiplicity distributions with $\eta_{c}=0.5$ are described by the single NBD [1]. Moreover, we also confirm that the GGL formula does work well for the explanation of the same data in present analyses. 
Table 1: Analysis of data on BEC by ALICE Collaboration and CMS Collaboration. Because estimated value of $1 / k^{(\mathrm{BEC})}$ is a unit, it is not cited.

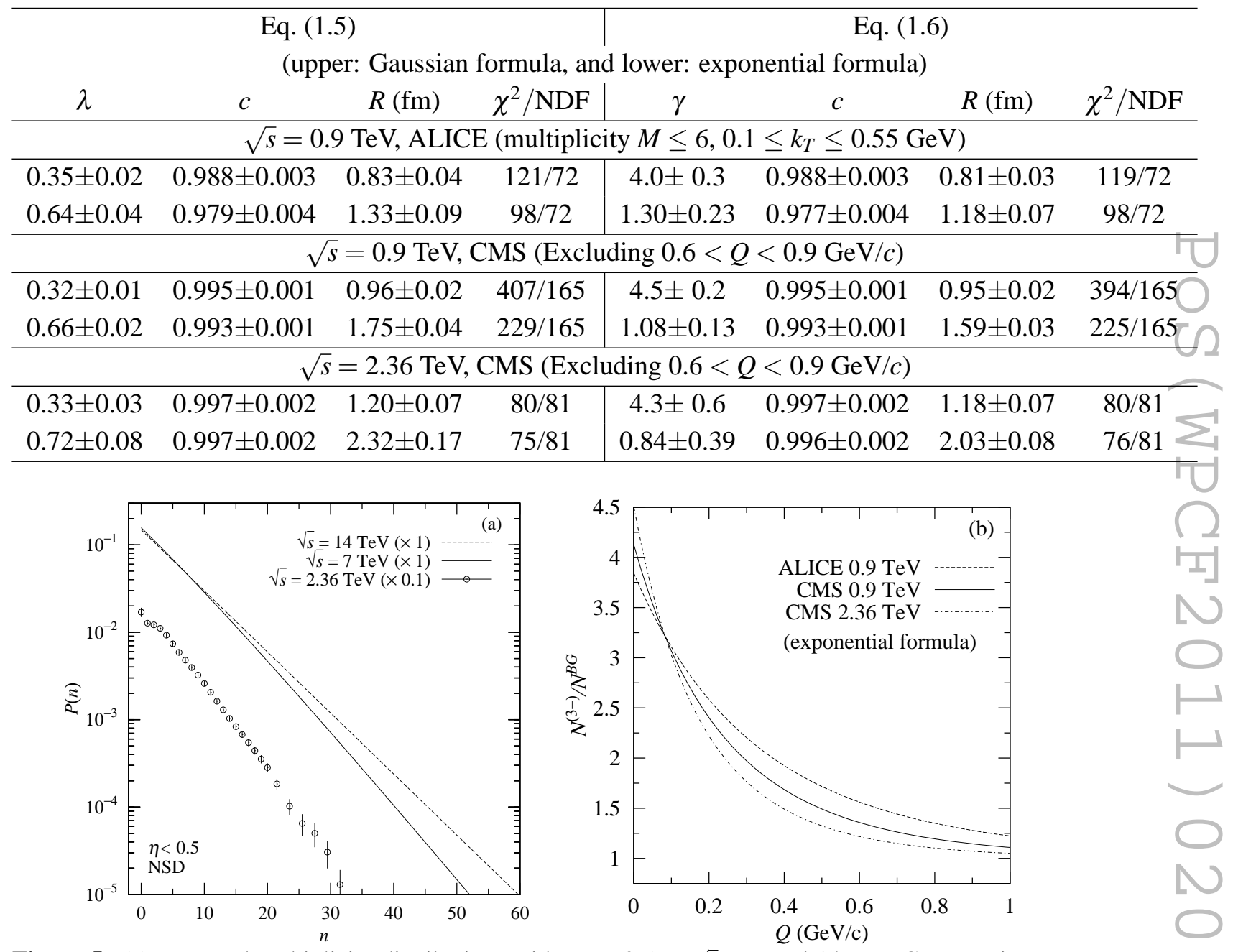

Figure 5: (a) Expected multiplicity distributions with $\eta_{c}=0.5$ at $\sqrt{s}=7$ and $14 \mathrm{TeV}$. Computations are based on the GGL formula (Eq. (1.3)) with values in Fig. 2 and $\langle n\rangle=2.5+0.76 \ln (\sqrt{s} / 0.2)$. (b) Our predictions of the $3 \mathrm{rd}$ order BEC at 0.9 and $2.36 \mathrm{TeV}$. Eq. (5.1) with values in Table 1 is used.

We observed that distributions with $\eta_{c}=0.5$ at $7 \mathrm{TeV}$ does not have the coherent component. In other words, the multiplicity distributions with $\eta_{c}=0.5$ at $7 \mathrm{TeV}$ are described by the NBD with $k=1$.

Using values in Fig. 2, we can predict multiplicity distributions with $\eta_{c}=0.5$ at 7 and $14 \mathrm{TeV}$ in Fig. 5a. Those are able to be examined in a near future. If there were discrepancies among data and predictions, we should consider the other effect, for example, due to the mini-jets [16].

Through present analyses of the BEC, results by the exponential formula seem to be better than those by the Gaussian formula in Table 1. See [17] for the source functions. Moreover, values of $\gamma$ 's obtained in Fig. 2 and Table 1 seem to be similar each other. To obtain more significant knowledge on the parameter $\gamma$, analyses of the multiplicity distributions and the BEC in the same hadronic ensembles are necessary[14, 15]. 
It is worthwhile to predict the $3 \mathrm{rd} \mathrm{BEC}$ at $0.9 \mathrm{TeV}$ using the same condition with $M \leq 6$, $0.1 \leq k_{T} \leq 0.55 \mathrm{GeV} / c$. Utilizing estimated values of $\gamma^{(\mathrm{BEC})}$ and $R$ in the 2 nd BEC by ALICE Collaboration, we can predict the 3rd order BEC; The following formula [14] is used,

$$
N^{(3-)} / N^{B G}=1+6 p(1-p) e^{-\frac{1}{3} R \sqrt{Q_{3}^{2}}}+3 p^{2}(3-2 p) e^{-\frac{2}{3} R \sqrt{Q_{3}^{2}}}+2 p^{3} e^{-R \sqrt{Q_{3}^{2}}},
$$

where $p=1 /(1+\gamma)$ and $Q_{3}^{2}=Q_{12}^{2}+Q_{23}^{2}+Q_{31}^{2}$. Our predictions on the 3rd order BEC at 0.9 and $2.36 \mathrm{TeV}$ are given in Fig. 5b. The results would be compared with measurements, as UA1 Minimum Bias Collaboration did [18]. By these comparisons, we could obtain more useful information on the parameter $\gamma$ and the role of the GGL formula.

Addendum: Recently CMS Collaboration has reported new analyses on BEC at 0.9 and $7 \mathrm{TeV}$ in $p p$ collisions [19]. We have applied Eq. (1.6) to data with the exponential form and the long range effect $(1+\alpha Q) . R=1.47 \mathrm{fm}(0.9 \mathrm{TeV})$ and $R=1.8 \mathrm{fm}(7 \mathrm{TeV})$ are obtained [20].

\section{References}

[1] K. Aamodt et al. [ALICE Collaboration], Eur. Phys. J. C 68 (2010) 89.

[2] G. J. Alner et al. [UA5 Collaboration], Phys. Lett. B 160 (1985) 193.

[3] R. E. Ansorge et al. [UA5 Collaboration], Z. Phys. C 43 (1989) 357.

[4] J. F. Grosse-Oetringhaus and K. Reygers, J. Phys. G 37 (2010) 083001.

[5] Ch. Fuglesang, Multiparticle Dynamics-Festschrift for Leon Van Hove and proceedings, La Thuile, Italy (1990)

[6] Z. Koba, H. B. Nielsen and P. Olesen, Nucl. Phys. B 40 (1972) 317.

[7] M. Biyajima, Prog. Theor. Phys. 69 (1983) 966 [Addendum-ibid. 70 (1983) 1468].

[8] M. Biyajima, Phys. Lett. 137B (1984) 225 [Addendum-ibid. 140B (1984) 435]; See also, M. Biyajima and N. Suzuki, Phys. Lett. B 143 (1984) 463.

[9] K. Aamodt et al. [ALICE Collaboration], Eur. Phys. J. C 68 (2010) 345.

[10] K. Aamodt et al. [ALICE Collaboration], Phys. Rev. D 82 (2010) 052001.

[11] V. Khachatryan et al. [CMS Collaboration], Phys. Rev. Lett. 105 (2010) 032001.

[12] T. Mizoguchi and M. Biyajima, Eur. Phys. J. C 70 (2010) 1061.

[13] G. Goldhaber, S. Goldhaber, W. Y. Lee and A. Pais, Phys. Rev. 120 (1960) 300.

[14] M. Biyajima, A. Bartl, T. Mizoguchi, O. Terazawa and N. Suzuki, Prog. Theor. Phys. 84 (1990) 931 [Addendum-ibid. 88 (1992) 157].

[15] R. M. Weiner, "Bose-Einstein correlations in particle and nuclear physics: A collection of reprints," Chichester, UK: Wiley (1997) 483 p; See, also G. Alexander, Rept. Prog. Phys. 66 (2003) 481.

[16] A. Giovannini and R. Ugoccioni, Phys. Rev. D 59 (1999) 094020 [Erratum-ibid. D 69 (2004) 059903].

[17] R. Shimoda, M. Biyajima and N. Suzuki, Prog. Theor. Phys. 89 (1993) 697.

[18] N. Neumeister et al. [UA1-Minimum Bias-Collaboration], Phys. Lett. B 275 (1992) 186; In Their analyses an exponential form should read as $r=R \sqrt{n(n-1) / 2}$ in higher order $n$.

[19] V. Khachatryan et al. [CMS Collaboration], JHEP 1105, 029 (2011).

[20] T. Mizoguchi and M. Biyajima, in preparation. 